\title{
BMJ Open Systematic review of preoperative physical activity and its impact on postcardiac surgical outcomes
}

\author{
D Scott Kehler, ${ }^{1,2}$ Andrew N Stammers, ${ }^{1,2}$ Navdeep Tangri, ${ }^{3}$ Brett Hiebert, ${ }^{4}$ \\ Randy Fransoo, ${ }^{5}$ Annette S H Schultz, ${ }^{6}$ Kerry Macdonald, ${ }^{7}$ \\ Nicholas Giacomontonio, ${ }^{8}$ Ansar Hassan, ${ }^{9}$ Jean-Francois Légaré, ${ }^{10}$ \\ Rakesh C Arora, ${ }^{4}$ Todd A Duhamel ${ }^{1,2}$
}

To cite: Kehler DS, Stammers AN, Tangri N, et al. Systematic review of preoperative physical activity and its impact on postcardiac surgical outcomes. BMJ Open 2017;7:e015712. doi:10.1136/ bmjopen-2016-015712

- Prepublication history and additional material for this paper are available online. To view these files please visit the journal online (http://dx.doi. org/10.1136/bmjopen-2017015712).

Received 22 December 2016 Revised 31 May 2017 Accepted 21 June 2017

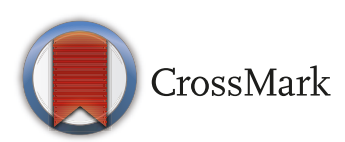

For numbered affiliations see end of article.

Correspondence to

Mr. D Scott Kehler;

SKehler@sbrc.ca

\section{ABSTRACT}

Objectives The objective of this systematic review was to study the impact of preoperative physical activity levels on adult cardiac surgical patients' postoperative: (1) major adverse cardiac and cerebrovascular events (MACCEs), (2) adverse events within 30 days, (3) hospital length of stay (HLOS), (4) intensive care unit length of stay (ICU LOS), (5) activities of daily living (ADLs), (6) quality of life, (7) cardiac rehabilitation attendance and (8) physical activity behaviour.

Methods A systematic search of MEDLINE, Embase, AgeLine and Cochrane library for cohort studies was conducted.

Results Eleven studies ( $n=5733$ patients) met the inclusion criteria. Only self-reported physical activity tools were used. Few studies used multivariate analyses to compare active versus inactive patients prior to surgery. When comparing patients who were active versus inactive preoperatively, there were mixed findings for MACCE, 30 day adverse events, HLOS and ICU LOS. Of the studies that adjusted for confounding variables, five studies found a protective, independent association between physical activity and MACCE $(n=1), 30$-day postoperative events $(n=2)$, HLOS $(n=1)$ and ICU LOS $(n=1)$, but two studies found no protective association for 30-day postoperative events $(n=1)$ and postoperative ADLs $(n=1)$. No studies investigated if activity status before surgery impacted quality of life or cardiac rehabilitation attendance postoperatively. Three studies found that active patients prior to surgery were more likely to be inactive postoperatively.

Conclusion Due to the mixed findings, the literature does not presently support that self-reported preoperative physical activity behaviour is associated with postoperative cardiac surgical outcomes. Future studies should objectively measure physical activity, clearly define outcomes and adjust for clinically relevant variables. Registration Trial registration number NCT02219815. PROSPER0 number CRD42015023606.

\section{INTRODUCTION}

Recent reports suggest that more than half of cardiac surgeries are being performed on older adults who are more likely to be frail
Strengths and limitations of this study

- There were mixed findings regarding the impact of physical activity on postcardiac surgical outcomes.

- Only self-reported physical activity tools were used

- The multiple tools to measure physical activity and the variety of definitions of outcomes did not allow for a quantitative synthesis (meta-analysis).

and have multiple comorbidities. ${ }^{1}$ While cardiac surgery has been shown to improve the outcomes of these patients, more than $75 \%$ of major perioperative complications and deaths occur in older adults. ${ }^{23}$ Before surgery, many of these patients are deconditioned and have diminished resilience in the face of major stressors such as cardiac surgery, and it has been postulated that they could benefit from a therapeutic intervention prior to their major surgical procedure in order to reduce their operative risk. However, little information exists to evaluate the benefit of preoperative risk reduction strategies for the older cardiac surgery patient.

Adopting and sustaining a more physically active lifestyle is typically intended to be a part of an interdisciplinary rehabilitation plan that is instituted postoperatively and has been shown to reduce the risk of cardiac mortality and hospital admissions and improve health-related quality of life (QOL) in patients. ${ }^{4}$ Importantly, older adults who sustain a physically active lifestyle after a postoperative exercise-based rehabilitation programme can continue to improve their functional walking status. ${ }^{5}$ However, evidence suggests that cardiac surgery patients are highly sedentary during the preoperative period, especially in older adults. ${ }^{6}$ Furthermore, few randomised controlled trials exist that evaluate the therapeutic benefit of preoperative lifestyle modification in patients 
undergoing cardiac surgery. ${ }^{7-9}$ Information regarding the link between preoperative physical activity and postoperative health outcomes in cardiac surgery patients would be valuable for healthcare providers to assist them in selecting patients who might benefit from preoperative exercise therapy.

The purpose of this systematic review was to compare the following postoperative outcomes between cardiac surgery patients defined as physically active prior to surgery and those who were defined as physically inactive preoperatively: (1) major adverse cerebrovascular and cardiovascular events (MACCEs), (2) 30-day adverse events as defined by the Society of Thoracic Surgeons (STS),$^{10}$ (3) hospital length of stay, (4) intensive care unit (ICU) length of stay, (5) health-related QOL, (6) activities of daily living (ADLs), (7) cardiac rehabilitation attendance and (8) physical activity levels postoperatively.

\section{MATERIAL AND METHODS}

The protocol for this systematic review has been described in PROSPERO: CRD42015023606. Note the following ad hoc changes to the previous protocol: ICU length of stay and postoperative physical activity as additional outcomes were explored in this systematic review.

\section{Eligibility criteria}

Eligible studies included cohort studies that examined adult (>18years) cardiac surgery patients undergoing coronary artery bypass grafting (CABG), aortic or mitral valve repair/replacement, transcatheter aortic valve implantation or combined procedures. Studies with patients undergoing congenital cardiac surgery, heart transplantation or left ventricular assist device implantation procedures were excluded. Studies could compare physically active versus inactive patients prior to cardiac surgery on the basis of subjective (eg, questionnaire) or objective (eg, pedometer and accelerometry) assessments of physical activity.

Eligible studies had to compare at least one of the following postoperative outcomes: MACCE defined as death, stroke, myocardial infarction and the need for emergency cardiac surgery; 30-day adverse events as defined by the STS, ${ }^{10}$ including an unexpected return to the operating room, complications due to pulmonary, cardiovascular, gastrointestinal, haematological, urological, infection, and neurological deficits, other important miscellaneous outcomes (eg, unexpected admission to ICU, or other events requiring admission to operating room requiring anaesthesia); hospital length of stay; ICU length of stay; health-related QOL with any assessment tool; ADLs using any evaluation strategy; cardiac rehabilitation attendance; and physical activity behaviour using either subjective or objective forms of assessment.

\section{Search strategy}

The search strategy was completed by a librarian and reviewed by a second librarian. The search included keywords and controlled vocabulary. English language limits were applied. Databases used included MEDLINE, Embase, AgeLine and Cochrane Library (CDSR, CENTRAL and DARE), and articles were searched from inception to December 2016. The MEDLINE strategy was registered and published online in PROSPERO (http://www.crd.york.ac.uk/PROSPEROFILES/23606_ STRATEGY_20150518.pdf) and is also available as a online supplementary file 1 . The search was validated through a cross-check of references of studies selected for inclusion. In addition, conference abstracts were hand searched using the internet. Attempts were made to contact authors of conference abstracts to determine if their findings were published in a peer-reviewed journal.

\section{Study selection}

The title, abstract and full-text article screening processes were independently completed by two reviewers. A training exercise for the title and abstract phase was conducted by the independent reviewers using a random sample of 100 titles and abstracts. Discrepancies in studies for inclusion were resolved by discussion of the two reviewers. The final observed agreement was $98 \%$ with a kappa statistic of 0.47 for the title and abstract screen. One training exercise of 10 randomly selected articles was completed for the fulltext screen. Discrepancies for inclusion were resolved through discussion. The observed agreement for the fulltext screen was $96 \%$ with a kappa statistic of 0.83 .

\section{Data abstraction}

Two reviewers independently extracted relevant data for the selected outcomes described above. Discrepancies in the data extraction procedure were resolved through discussion. Data abstraction items included study characteristics (eg, authors, year of publication, sample size and follow-up time points if relevant), patient characteristics (eg, age, sex and surgery type), physical activity tool used and the outcomes that were measured.

\section{Risk of bias assessment}

Two reviewers independently reviewed the risk of bias of each included study using the Newcastle-Ottawa Scale. ${ }^{11}$ Items within this tool assess the risk of bias associated with selection of participants, comparability (eg, study authors controlled for patient demographics and clinical characteristics) and outcome assessment (eg, data collection method for outcome, sufficient follow-up and adequacy of follow-up of cohorts). Each study was given a score within each category (selection: 0-4, comparability: 0-2 and outcome: $0-3$ ) and an overall score ranging from 0 to 9 . A score of 0 suggests an increased risk of bias and a higher score suggests a lower risk of bias.

\section{Quantitative synthesis}

Due to the significant heterogeneity between studies in terms of physical activity assessment tools used and outcomes assessed, meta-analyses were not performed. 


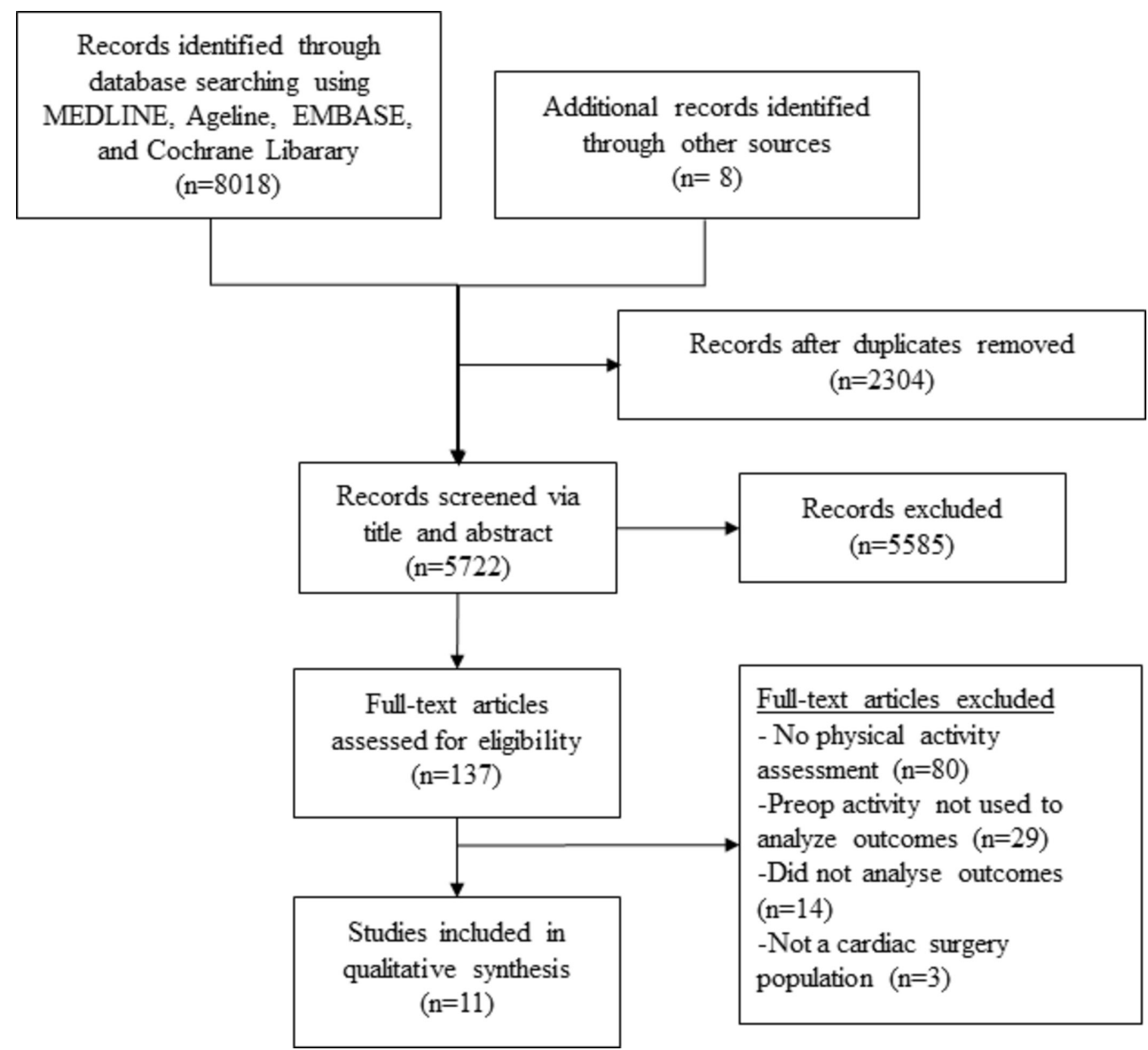

Figure 1 Study flow diagram.

\section{RESULTS}

The literature search results are shown in figure 1. After removing duplicates, 5722 articles were title and abstract screened. A total of 137 articles were then assessed in full text. Eleven studies met the eligibility criteria for the final analysis, and they included a total of 5733 patients. ${ }^{12-22}$

An overview of the included studies can be viewed in table 1 . In the studies by Markou et al, ${ }^{1214}$ Nery et $a l^{1316}$ and Martini et al, ${ }^{15}$ they evaluated CABG-only patients. Rengo et al, ${ }^{17}$ Giaccardi et al, ${ }^{18}$ Cacciatore et al, ${ }^{19}$ Noyez et $a l^{20}$ and Min $e t a l^{21}$ evaluated both CABG and/or valve procedures, and van Laar $e t a l^{22}$ evaluated isolated aortic valve repair patients. The average age of participants in different studies ranged from 60 years (Martini and Nery et $a l^{1516}$ ) to 75 years (Rengo et al, Giaccardi et al, Min et al, van Laar et al ${ }^{17182122}$ ). Rengo et al, ${ }^{17}$ Giaccardi et al, ${ }^{18}$ Min et $a t^{21}$ and van Laar $e t a t^{22}$ excluded patients with physical impairments or with New York Heart Association heart failure class IV symptoms (severe cardiac symptoms), but in general exclusion criteria were not explicitly reported. Studies were conducted in the Netherlands (Markou $e t$ $a l,{ }^{12}{ }^{14}$ Noyez $e t a l^{20}$ and van Laar $e t a l^{22}$ ), Brazil (Nery $e t$ $a l^{1316}$ and Martini et $a l^{15}$ ), Italy (Rengo et $a l^{17}$ Giaccardi $e t a l^{18}$ and Cacciatore $e t a l^{18}$ ) and the USA (Min $e t a l^{21}$ ). Two studies by Nery et $a l^{13}$ and Martini et $a l^{15}$ used the same patient sample but examined different outcomes.
The sample size of studies ranged from 35 in the Min $e t$ $a l^{21}$ study to 3150 in the Noyez et $a l^{20}$ study.

\section{Physical activity tools}

The physical activity assessments in each study were based on self-reported assessment tools. The timing of the physical activity assessments prior to surgery was not reported by Cacciatore et al ${ }^{19}$ Nery et $a l^{13}{ }^{16}$ Markou et $a l^{1214}$ or Martini $e t a l .{ }^{15}$ Rengo $e t a l^{17}$ reported the timing of their physical activity assessment, which was within $35 \pm 6$ days prior to surgery. Noyez et al and van Laar et al measured activity the day before surgery. ${ }^{20}{ }^{22}$ Min and colleagues measured physical activity 4 weeks prior to the patients' surgical procedure. ${ }^{21}$ Finally, Giaccardi et al measured preoperative physical activity levels approximately 1 week following surgery. ${ }^{18}$

Four studies used the Corpus Christi Heart Project questionnaire, ${ }^{12} 142022$ which asks participants about their typical physical activity behaviours over the past year during their leisure time. Participants were categorised into a sedentary group if they accumulated less than 30 min per day of light intensity activity, or into an active group if they accumulated at least one session per week of dynamic activity lasting $\geq 15$ min marked by moderate intensity. Nery et $a l^{1316}$ and Martini et $a l^{15}$ used a structured questionnaire confirmed by the Minnesota Leisure 


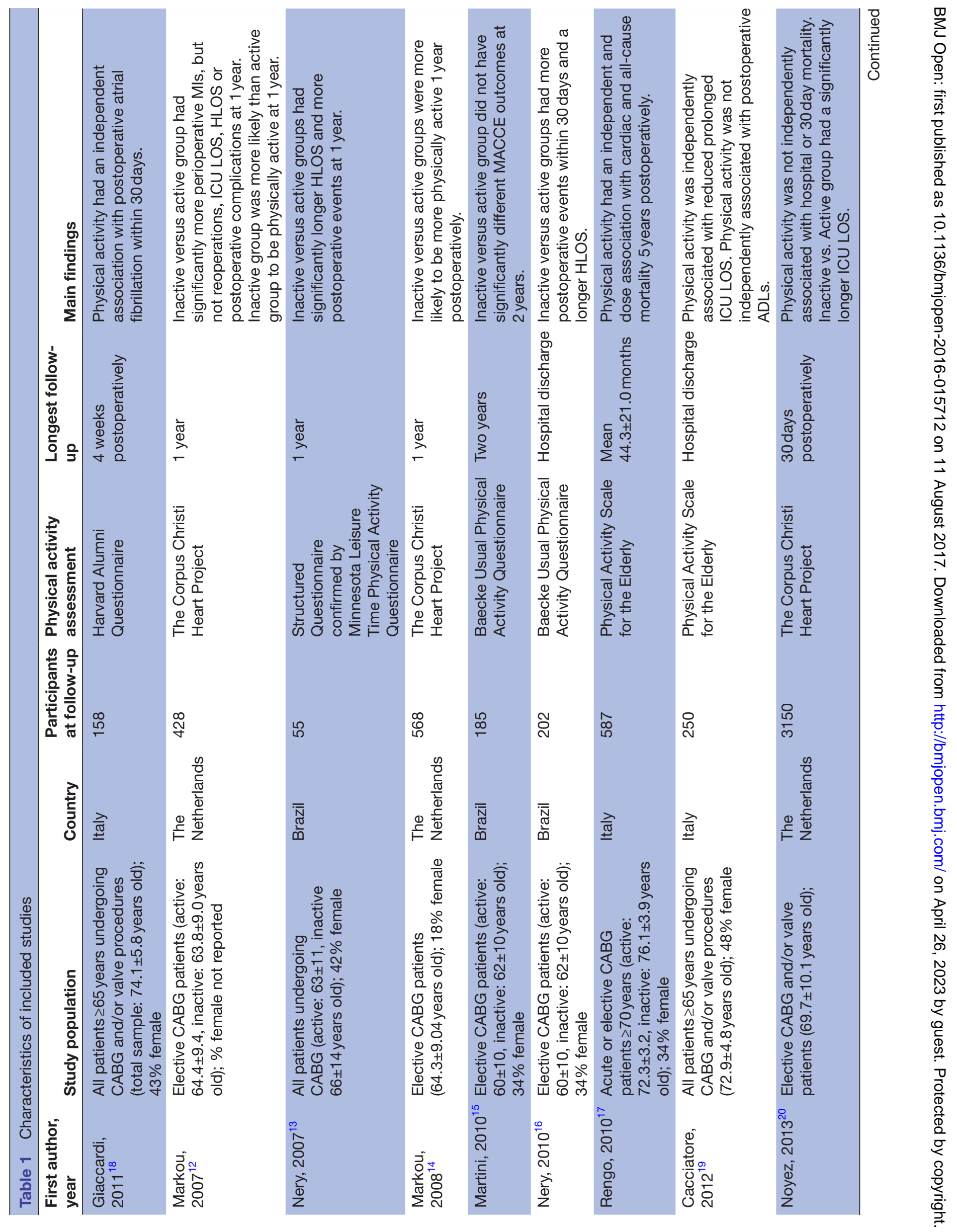


Time Physical Activity Questionnaire ${ }^{13}$ or the Baecke Usual Physical Activity Questionnaire. ${ }^{15} 16$ Both physical activity tools ask participants to recall their usual activities 12 months prior and determine the frequency, intensity and time of activity. Participants were categorised into an inactive group if they engaged only in light intensity $(<3$ metabolic equivalents) activity or into an active group if they achieved $\geq 3$ metabolic equivalents. Rengo et $a l^{17}$ and Cacciatore et $a l^{19}$ used the Physical Activity Scale for the Elderly, which is a 7-day recall of a participant's frequency, intensity, duration and type of activity. Participants receive a total score from 0 to 400. Rengo et $a l^{17}$ separated participants by inactive and active groups using the median score, whereas Cacciatore $e t a l^{9}$ used the continuous measure. The Harvard Alumni Questionnaire was implemented by Giaccardi and colleagues, ${ }^{18}$ which measures the typical weekly amount and intensity of physical activity over the past year. Participants were categorised as inactive if they participated in $<1$ hour per week of light activity and as active if they participated in either $\geq 4$ hours of light or more than 1-2hours of moderate activity per week. In the study by Min $e t a l^{21}$ the physical activity-related questions were used from the Health and Retirement Survey, which determines a participant's frequency and intensity of activity in a typical week. These authors used the continuous score in their study.

\section{MAJOR ADVERSE CARDIAC AND CEREBROVASCULAR EVENTS}

Outcomes within the definition of MACCE were evaluated in four studies (table 2) by Nery et al, ${ }^{13}$ Martini et al, ${ }^{15}$ Rengo $e a^{17}$ and van Laar $e t a l^{22}$ The follow-up periods were one (Nery et $a l^{13}$ ), two (Martini et $a l^{15}$ and van Laar et $a l^{22}$ ) and 5 years (Rengo et $a l^{17}$ ) postoperatively. Unadjusted differences between active versus inactive patients and MACCE (defined as atrial fibrillation, hospital admission, reoperation and myocardial infarction) were found 1 year postoperatively in the Nery $e t a l^{13}$ study. The Martini et $a l^{15}$ study found no differences (defined as mortality, rehospitalisation, cerebrovascular accident and MI) at 2 years postoperatively. The unadjusted rates of mortality within 2years postsurgery were significantly lower in the active versus inactive group in the study by van Laar and colleagues. ${ }^{22}$ The study by Rengo and colleagues found a significant and dose-response relationship between physical activity and postoperative cardiac and all-cause mortality after controlling for preoperative demographics, medical history, medications and clinical characteristics. ${ }^{17}$

\section{0-day events}

Five studies (Markou et al, ${ }^{12}$ Nery et al, ${ }^{16}$ Rengo et al, ${ }^{17}$ Giaccardi et $a l^{18}$ and Noyez et $a l^{20}$ ) evaluated postoperative events within 30 days of surgery (table 2). The postoperative events measured varied significantly between the studies. Three studies (Nery et al, ${ }^{16}$ Giaccardi et $a l^{18}$ and Noyez et $a l^{20}$ ) examined if physical activity was an 


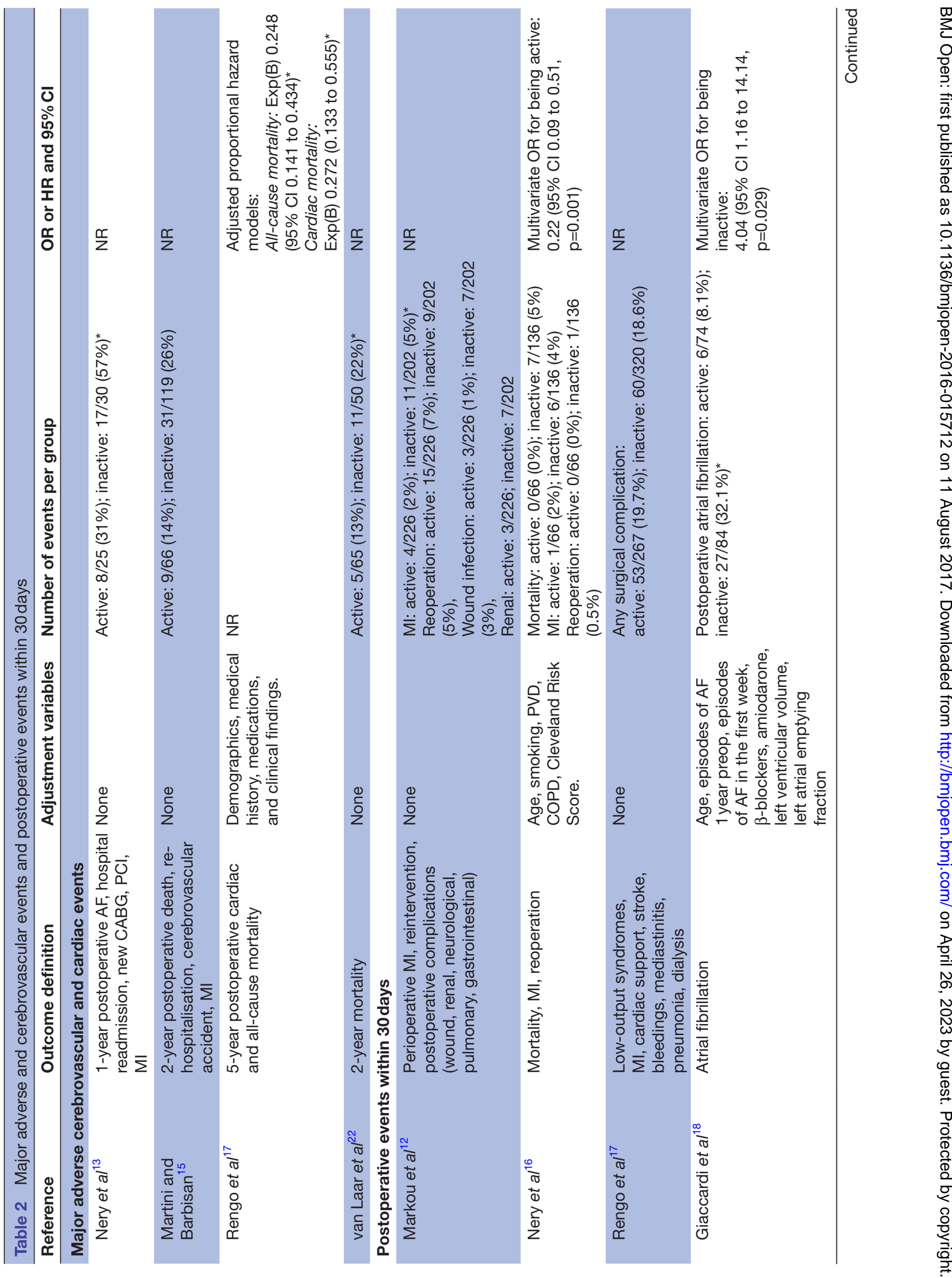




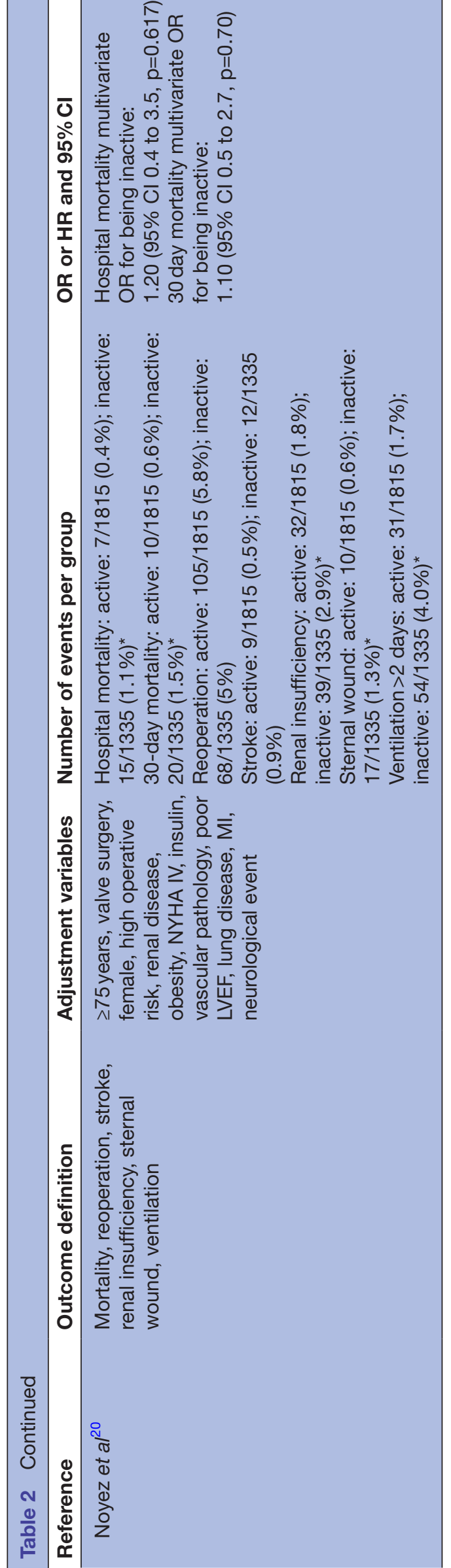

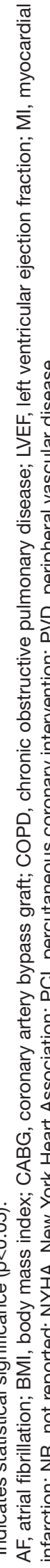

independent protective factor against postoperative events. Physical activity was an independent protective factor against the combined outcome of mortality, MI and reoperation in the study by Nery et $a l^{16}$ as well as postoperative atrial fibrillation in the Giaccardi and colleagues study ${ }^{18}$ but was not significant for in-hospital or 30-day mortality in the Noyez et $a l^{20}$ study.

\section{Postoperative health-related QOL}

No studies evaluated postoperative health-related QOL.

\section{Hospital and ICU length of stay}

Three studies by Markou et $a l^{12}$ and Nery et $a l^{13} 16$ compared hospital length of stay between active and inactive cardiac surgery patients (table 3). Hospital length of stay was longer in the inactive group in two of three studies (both by Nery et al). ${ }^{13}{ }^{16}$ One of the studies by Nery et $a l^{16}$ did not report hospital length of stay summary statistics between the active and inactive groups. However, that study reported an independent association between the preoperative active and inactive group and a reduced likelihood of prolonged hospital length of stay, though 'prolonged' was not defined in the study.

Three studies compared ICU length of stay between the preoperative physical activity groups (table 3) (Markou et $a l,{ }^{12}$ Cacciatore $e t a l^{19}$ and Noyez et al). ${ }^{20}$ Two studies (Markou et $a l^{12}$ and Noyez et $a l^{20}$ ) found that the inactive group had a significantly longer ICU length of stay compared with the active group. In the study by Cacciatore and colleagues, they found in their multivariate analysis that the active group was less likely to have a prolonged ICU length of stay $>3$ days compared with the inactive group after controlling for age, off-pump CABG, stroke and renal failure.

\section{Postoperative ADLS}

One study by Min $e t a l^{19}$ examined the impact of preoperative physical activity and postoperative ADLs at the time of hospital discharge and revealed no statistically significant $(p=0.079)$ association between the two after adjusting for preoperative demographics and clinical variables.

\section{Cardiac rehabilitation attendance}

No studies evaluated cardiac rehabilitation attendance postoperatively.

\section{Postoperative physical activity behaviour}

The impact of preoperative physical activity on postoperative physical activity levels was examined in the two studies by Markou et $a l^{1214}$ and in the other study by Min $e t a l^{21}$ (table 3). These studies found that the active group preoperatively was more likely to be physically inactive postoperatively. In both of the Markou et $a l^{1214}$ studies, they completed a multivariate analyses and found that this association remained statistically significant after controlling for age, gender and preoperative clinical characteristics. 


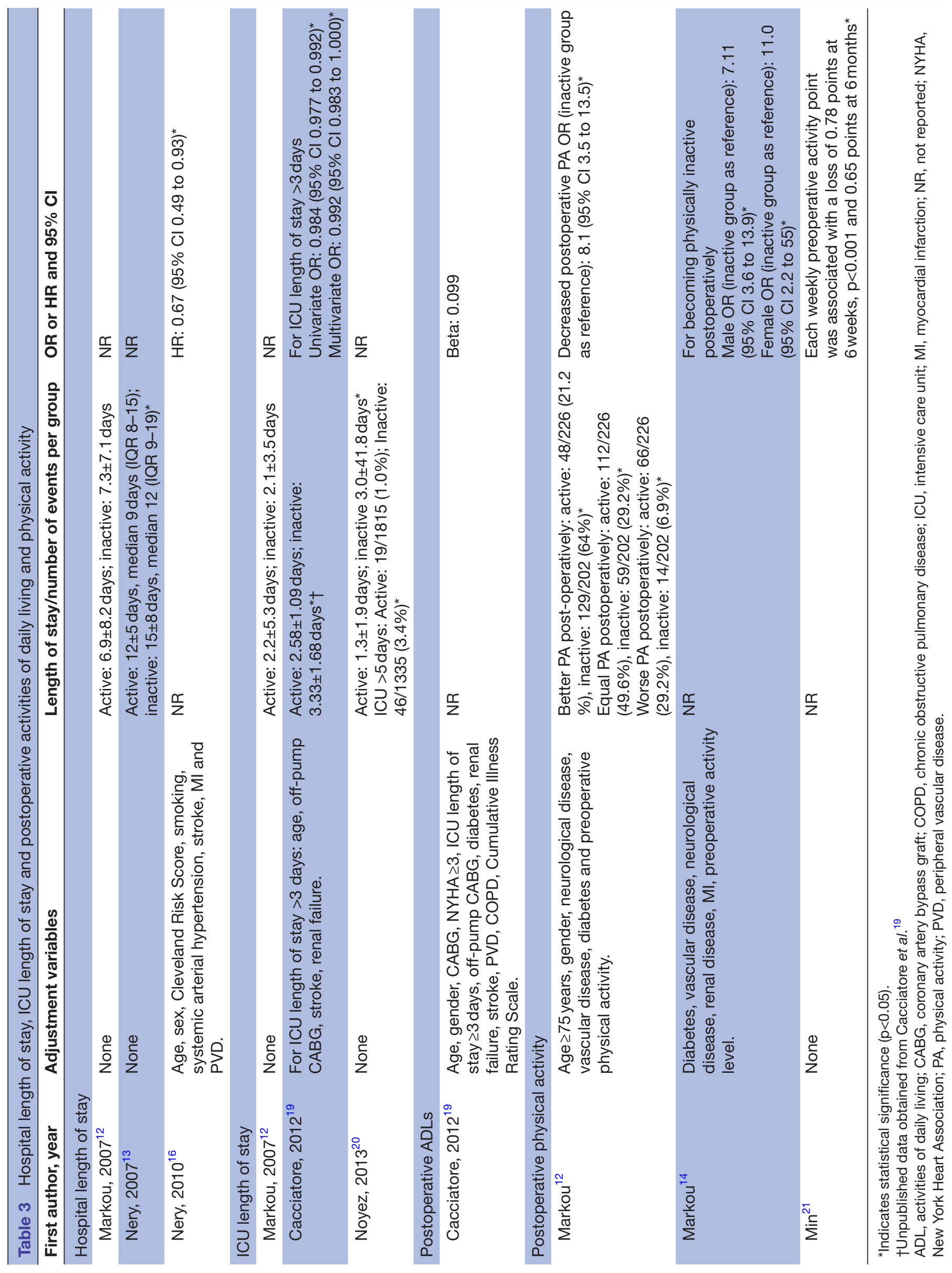

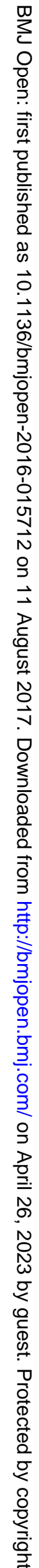


Table 4 Newcastle-Ottawa Scale risk of bias scores

\begin{tabular}{|c|c|c|c|c|}
\hline Reference & Selection & Comparability & Outcome & Total \\
\hline Markou et $a l^{12}$ & 3 & 2 & 3 & 8 \\
\hline Nery et al ${ }^{13}$ & 3 & 0 & 2 & 5 \\
\hline Martini and Barbisan ${ }^{15}$ & 3 & 0 & 2 & 5 \\
\hline Nery and Barbisan ${ }^{16}$ & 3 & 2 & 2 & 7 \\
\hline Giaccardi et $a l^{18}$ & 3 & 2 & 2 & 7 \\
\hline Cacciatore et $\mathrm{al}^{19}$ & 3 & 2 & 2 & 7 \\
\hline Noyez et $a l^{20}$ & 3 & 2 & 3 & 8 \\
\hline Min et $a l^{21}$ & 4 & 2 & 1 & 7 \\
\hline van Laar et $a^{22}$ & 3 & 0 & 3 & 6 \\
\hline
\end{tabular}

Maximum scores are 4, 2 and 3 for selection, comparability and outcome, respectively. Maximum total score is 9 . A lower score within each category and for a total score indicates a higher risk of bias.

\section{Risk of bias}

The risk of bias assessment via the Newcastle-Ottawa Scale can be viewed in table 4 . Since some studies assessed multiple outcomes, the risk of bias assessments were based on their highest possible score (eg, some outcomes were assessed with a multivariable analysis, while others were not in the same study). All studies scored at least 3 out of 4 for the selection of study groups. There was variability across studies for the ascertainment of exposure or outcome of interest. Total risk of bias scores ranged from 5 to 9 , suggesting the studies were of moderate to high quality, respectively.

\section{DISCUSSION}

The purpose of this systematic review was to determine if physical activity before cardiac surgery was associated with postoperative health outcomes. Given the different self-reported physical activity tools used that prevented comparison across studies, the inconsistent use of adjustment for potential confounders, and the varying outcomes evaluated for MACCE and 30-day postoperative events, it cannot be concluded that preoperative physical activity is associated with postoperative outcomes in adult cardiac surgery patients. This systematic review highlights important gaps within the literature on this topic. Therefore, key recommendations for examining the impact of preoperative physical activity behaviour on postsurgical outcomes of cardiac patients are provided (table 5).

The different self-reported physical activity tools used across the studies makes it difficult to compare the preoperative physical activity levels of patients prior to cardiac surgery. Even so, it is important to note that in the studies included in this systematic review, most of the studies identified a subsample of cardiac surgery patients who were more vulnerable to poor health outcomes by categorising patients as active or inactive prior to surgery using their self-reported physical activity measures. However, the way the physical activity tools measured physical activity (eg, over the past year or in the past week; see the Methods section) could have influenced the outcomes of the study. There seems to be no universally accepted tool to measure self-reported physical activity levels, ${ }^{23}$ and it is unclear if any of the physical activity tools identified by this review have been validated in the cardiac surgery patient. One advantage of using self-reported physical activity measures in studies is their ease of administration compared with other objectively measured physical activity tools. Furthermore, self-reported physical activity tools appear to provide some value when assessing the independent association between activity levels and poor outcomes. In fact, most physical activity guideline recommendations for health benefits, including those in North America, are based on self-reported measures. ${ }^{24}$ Another strength of using a subjective physical activity tool in the preoperative cardiac surgery patient is that it would capture a patient's physical activity behaviour before they are placed on a waiting list, when they might refrain from being physically active in fear of making their condition worse. However, cardiac surgery patients and other patient populations tend to misreport their physical activity levels compared with objectively measured physical activity. ${ }^{626}$ Nevertheless, this systematic review found no studies that evaluated objectively measured physical activity before cardiac surgery and its link to postoperative health outcomes. Evidence suggests there is a stronger association between objective measures of physical activity and various cardiovascular and metabolic biomarkers as compared with subjective measures of physical activity. ${ }^{27}{ }^{28}$ While it is unclear which objective measures of physical activity are most appropriate in the complex cardiac surgical patients, future studies should use a physical activity tools such as accelerometers or pedometers. 
Table 5 Guidelines for physical activity measurement and outcome assessment in cardiac surgery patients: limitations and opportunities for future research

\begin{tabular}{ll}
\hline Drawbacks & Opportunity \\
\hline Physical activity & Use of objectively measured tools (eg, pedometers and accelerometers) accompanied \\
1. Heterogeneity in tools used & by a questionnaire that can produce data that can be compared across studies, such \\
across studies & as step counts, intensity and duration of physical activity. \\
2. Only subjective measures were & Capture physical activity behaviour as soon as a patient is placed on a wait list, or in \\
used & non-elective cases, as soon as possible prior to surgery. \\
3. Time of preoperative physical & Physical activity should be assessed ideally over a 7-day period. \\
activity assessment was unclear & Physical activity should be assessed by intensity and duration per week and in steps \\
in most studies & per day. \\
& MACCE should be evaluated as a long-term outcome and defined as death, stroke, \\
Outcomes & myocardial infarction and the need for redo cardiac surgery. Each outcome should be \\
4. Heterogeneity in MACCE & evaluated individually. \\
and postoperative events within & 30-day postoperative events should be evaluated using the STS checklist ${ }^{10}$ : along \\
30 days definitions & with reasons, evaluate unexpected return to the operating room, complications due \\
& to pulmonary, cardiovascular, gastrointestinal, haematological, urological, infection, \\
& neurological and other important miscellaneous outcomes (eg, unexpected admission \\
& to ICU, or other events requiring admission to operating room requiring anaesthesia. \\
& Rehospitalisation for any cause after cardiac surgery should also be added to \\
& outcomes.
\end{tabular}

ICU, intensive care unit; MACCE, major adverse cerebrovascular and cardiac events; STS, Society of Thoracic Surgeons.

There were inconsistent findings across studies assessing the same outcomes, and many studies did not adjust for clinically relevant variables that could influence the health outcomes of cardiac surgery patients. It is possible that most of the included studies were not statistically powered to detect changes between inactive and active groups. The study by Rengo et $a l^{17}$ had the largest sample size of the four studies that assessed MACCE outcomes, which found a significant protective association between preoperative physical activity and cardiac and all-cause mortality 5 years postoperatively after controlling for clinically relevant variables (table 2). In contrast, the largest study examined in this systematic review by Noyez and colleagues ${ }^{20}$ found no association between preoperative activity and hospital and 30-day mortality after controlling for covariates (table 2). It is difficult to determine if patient-level factors influence outcomes (eg, elective or acute patients, surgery type, older vs younger, females vs males) as the samples were somewhat heterogeneous. Even so, some of the results of this systematic review are promising. Specifically, of the studies that controlled for confounding variables, five studies found a protective, independent association between higher preoperative physical activity levels when assessing clinical outcomes, including MACCE,${ }^{17} 30$-day postoperative events, ${ }^{16}{ }^{18}$ hospital length of stay ${ }^{16}$ and ICU length of stay, ${ }^{19}$ whereas only two studies found no protective association for 30-day postoperative events ${ }^{20}$ and postoperative ADLs. ${ }^{19}$ Yet, more studies are needed to elucidate the impact of preoperative physical activity on postcardiac surgical outcomes that control for clinically relevant variables. Clinical variables included in the cardiac surgical risk models (eg, EuroSCORE and STS score) could attenuate or mitigate the relationship between preoperative physical activity behaviour and postoperative outcomes. Collectively, future studies are needed to determine if preoperative physical activity is a protective factor for health outcomes after cardiac surgery that control for clinically relevant variables known to impact cardiac surgery outcomes.

An unanticipated finding was that patients who were active before surgery had a higher likelihood of being physically inactive postoperatively, after controlling for comorbidities. ${ }^{12} 1421$ Healthcare providers may have advised patients with more severe symptomology prior to surgery to refrain from physical activity. Also, the relief of cardiac symptoms after surgery among inactive patients 
could have led them to become more active postoperatively. However, these possibilities were not explored in the included studies.

While outside the scope of this systematic review, future studies should investigate if changes to physical activity levels prior to cardiac surgery impact long-term patient health outcomes. Cardiac rehabilitation programmes are intended to support cardiac patients in becoming more physically active postoperatively, and it has been shown that patients who attend such programmes reduce their risk for cardiac-related mortality and hospitalisation rates. ${ }^{29}$ Evidence suggests that among those referred to cardiac rehabilitation after cardiac surgery, only $40 \%$ attend. ${ }^{6}$ However, the literature is less clear on whether patients who attend cardiac rehabilitation are more physically active compared with those who do not attend. It is possible that patients who adopt and sustain a more physically active lifestyle on their own after cardiac surgery could yield similar health benefits compared with those who attend an exercise-based rehabilitation programme, but this hypothesis requires further investigation.

Previous randomised controlled trials comparing an exercise programme to standard care prior to elective cardiac surgery (ie, 'Prehab') demonstrate reductions in hospital length of stay and improvements in walking ability postoperatively. ${ }^{7-9}$ However, there were mixed findings from this systematic review when comparing preoperative physical activity behaviour and hospital stay. ${ }^{12} 16$ These divergent findings suggest either that a medically supervised and individualised physical activity programme is needed to derive the health benefits of physical activity prior to cardiac surgery, or that patients are misreporting their physical activity behaviours. Future cohort studies in this area should address the drawbacks of the included studies in this systematic review included in table 5 , while randomised trials should focus on whether preoperative exercise therapy programmes are feasible and efficacious in clinical practice.

The findings of this systematic review suggest that the literature would benefit from standardisation of the definition of measures such as MACCE and postoperative events within 30 days. The heterogeneity in reporting of outcomes can lead to considerably different conclusions across studies. ${ }^{30}$ Attempts should also be made to ensure other clinically important outcomes are captured, such as the addition of 30 day events. Only one study in this review compared physically active versus inactive patients preoperatively and reported on the individual postoperative events within 30 days. ${ }^{20}$ Collectively, uniform outcome reporting and appropriate outcome definitions are recommended when examining the outcomes of cardiac surgery. ${ }^{30}$

Patient-oriented outcomes should also be captured to ensure that cardiac surgery is improving other outcomes that patients value. No studies in this review determined if there was a link between preoperative physical activity behaviour and postoperative health-related QOL, and only one study evaluated postoperative ADLs. ${ }^{19}$ QOL postoperatively tends to improve in some older patients, while others tend to decline. ${ }^{31}$ Importantly, the preoperatively physical activity and overall functional status of cardiac surgery patients could play a role in the postoperative trajectory of these outcomes such as QOL. Other patient-oriented outcomes, including postoperative pain and cardiac symptoms, could also be investigated.

If physical activity is to be assessed in the preoperative period, the extent of missing data may also be a concern, especially with objective physical activity measures. The possibility of missing data from individual studies included in this systematic review was outside the objectives of the present study but is a salient point that should be considered for future investigations. It is also important to understand patient-level factors associated with missing data. The use of statistical techniques that address missing data, such as multiple imputation, is one approach to address missing physical activity data. Importantly, it has been shown that multiple imputation leads to precise estimates of predicting 30-day mortality risk in cardiac surgery patients when important clinical variables are missing, as compared with estimating risk with a complete case analysis. ${ }^{32}$

\section{Limitations}

One limitation to consider is that the patients included across the studies evaluated in this systematic review may have been different, as the recruitment criteria were not always clearly stated. A small sample of studies explicitly stated that they excluded those with physical limitations and healthcare providers may have advised higher risk patients to not participate in physical activity. There is also a limitation associated with the methodology of this systematic review: only studies written in English were included, raising the possibility that some studies were missed.

\section{CONCLUSION}

Due to the mixed findings in this systematic review, it cannot be concluded that self-reported physical activity behaviour before cardiac surgery is associated with health outcomes after surgery. The mixed findings could be due to the heterogeneity in physical activity tools used, definitions of outcomes and the few studies adjusting for other potentially confounding variables. These findings highlight the need for more research in this area.

\section{Author affiliations}

${ }^{1}$ Health, Leisure \& Human Performance Research Institute, Faculty of Kinesiology and Recreation Management, University of Manitoba, Winnipeg, Manitoba, Canada

${ }^{2}$ Institute of Cardiovascular Sciences, St. Boniface Hospital Research Centre, Winnipeg, Canada

${ }^{3}$ Seven Oaks Hospital Research Centre, Winnipeg, Canada

${ }^{4}$ Department of Surgery, University of Manitoba and Cardiac Sciences Program, Winnipeg, Canada

${ }^{5}$ Department of Community Health Sciences and Manitoba Centre for Health Policy, University of Manitoba, Winnipeg, Canada

${ }^{6}$ College of Nursing, Faculty of Heath Sciences, University of Manitoba, Winnipeg, Canada 
${ }^{7}$ Seven Oaks Hospital Library, Winnipeg, Canada

${ }^{8}$ Division of Cardiology, Department of Medicine, Dalhousie University, Halifax, Canada

${ }^{9}$ Department of Cardiac Surgery, New Brunswick Heart Centre, Saint John Regional Hospital, Saint John, Manitoba, Canada

${ }^{10}$ Division of Cardiac Surgery, Department of Surgery, Dalhousie University, Halifax, Canada

Acknowledgements Dr Francesco Cacciatore and his research group provided additional data on ICU length of stay. TAD was supported by a Manitoba Health Research Council (MHRC) Establishment Grant. DSK was supported by an MHRC Studentship, a CIHR Frederick Banting and Charles Best Canada Graduate Scholarship, a CIHR Strategic Training Initiative in Health Research (STIHR) Knowledge Translation Canada Student Fellowship, a CIHR STIHR Population Intervention for Chronic Disease Prevention Fellowship and the Heart and Stroke Foundation Dr. Dexter Harvey Award. ANS was supported by a CIHR Canada Graduate Scholarship.

Contributors DSK was responsible for (1) analysis and interpretation of data, (2) drafting and revising the manuscript and (3) consenting for manuscript submission. ANS, BH, NT, RF, ASHH, NG, AH, J-FL were responsible for (1) analysis and interpretation of data, (2) revising the manuscript and (3) consenting for manuscript submission. KM was responsible for (1) developing the systematic review literature search, (2) analysis and interpretation of data, (3) drafting and revising the manuscript and (4) consenting for manuscript submission. RCA and TAD were responsible for (1) the conception and design, and analysis and interpretation of data, (2) revising the manuscript and (3) consenting for manuscript submission. RCA and TAD are cosenior authors.

Funding The work was supported by an Operating Grant from the Canadian Institutes of Health Research.

Competing interests None declared.

Provenance and peer review Not commissioned; externally peer reviewed.

Data sharing statement There are no additional unpublished data to share as a part of this systematic review.

Open Access This is an Open Access article distributed in accordance with the Creative Commons Attribution Non Commercial (CC BY-NC 4.0) license, which permits others to distribute, remix, adapt, build upon this work non-commercially, and license their derivative works on different terms, provided the original work is properly cited and the use is non-commercial. See: http://creativecommons.org/ licenses/by-nc/4.0/

(c) Article author(s) (or their employer(s) unless otherwise stated in the text of the article) 2017. All rights reserved. No commercial use is permitted unless otherwise expressly granted.

\section{REFERENCES}

1. Ferguson TB, Hammill BG, Peterson ED, et al. A decade of change-risk profiles and outcomes for isolated coronary artery bypass grafting procedures, 1990-1999: a report from the STS National Database Committee and the Duke clinical Research Institute. Society of Thoracic Surgeons. Ann Thorac Surg 2002;73:480-9.

2. Pfisterer M, Buser P, Osswald $S$, et al. Outcome of elderly patients with chronic symptomatic coronary artery disease with an invasive vs optimized medical treatment strategy: one-year results of the randomized TIME trial. JAMA 2003;289:1117-23.

3. Alexander KP, Anstrom KJ, Muhlbaier LH, et al. Outcomes of cardiac surgery in patients $>$ or $=80$ years: results from the National Cardiovascular Network. J Am Coll Cardiol 2000;35:731-8.

4. Anderson L, Oldridge N, Thompson DR, et al. Exercise-Based Cardiac Rehabilitation for Coronary Heart disease: cochrane Systematic Review and Meta-Analysis. J Am Coll Cardiol 2016;67:1-12.

5. Macchi C, Polcaro P, Cecchi F, et al. One-year adherence to exercise in elderly patients receiving postacute inpatient rehabilitation after cardiac surgery. Am J Phys Med Rehabil 2009;88:727-34.

6. Horne D, Kehler DS, Kaoukis G, et al. Impact of physical activity on depression after cardiac surgery. Can J Cardiol 2013:29:1649-56.

7. Arthur HM, Daniels C, McKelvie R, et al. Effect of a preoperative intervention on preoperative and postoperative outcomes in low-risk patients awaiting elective coronary artery bypass graft surgery. A randomized, controlled trial. Ann Intern Med 2000;133:253-62.

8. Sawatzky JA, Kehler DS, Ready AE, et al. Prehabilitation program for elective coronary artery bypass graft surgery patients: a pilot randomized controlled study. Clin Rehabil. 2014;28:648-57.

9. Herdy AH, Marcchi PL, Vila A, et al. Pre- and postoperative cardiopulmonary rehabilitation in hospitalized patients undergoing coronary artery bypass surgery: a randomized controlled trial. Am J Phys Med Rehabil 2008;87:714-9.

10. Society of Thoracic Surgeons. General Thoracic Surgery Database http://www.sts.org/sts-national-database/database-managers/ general-thoracic-surgery-database (accessed 17 Feb 2015).

11. Wells GA, Shea B, O'Connell D, et al. The Newcastle-Ottawa Scale (NOS) for assessing the quality of nonrandomised studies in metaanalyses. $2011 \mathrm{http}: / /$ www.ohri.ca/programs/clinical_epidemiology/ oxford.asp (accessed 25 Jan 2015).

12. Markou AL, Lasten PJ, Noyez L. Physical activity post myocardial revascularization. "will surgery improve my mobility? J Cardiovasc Surg 2007;48:201-6.

13. Nery RM, Barbisan JN, Mahmud MI. Influence of the practice physical activity in the coronary artery bypass graft surgery results. Rev Bras Cir Cardiovasc 2007;22:297-302.

14. Markou AL, Evers M, van Swieten HA, et al. Gender and physical activity one year after myocardial revascularization for stable angina. Interact Cardiovasc Thorac Surg 2008;7:96-101.

15. Martini MR, Barbisan JN. Influence of physical activity during leisure time in patients in the follow-up two years after CABG. Rev Bras Cir Cardiovasc 2010;25:359-64.

16. Nery RM, Barbisan JN. Effect of leisure-time physical activity on the prognosis of coronary artery bypass graft surgery. Rev Bras Cir Cardiovasc 2010;25:73-8.

17. Rengo G, Galasso G, Vitale DF, et al. An active lifestyle prior to coronary surgery is associated with improved survival in elderly patients. J Gerontol A Biol Sci Med Sci 2010;65:758-63.

18. Giaccardi M, Macchi C, Colella A, et al. Postacute rehabilitation after coronary surgery: the effect of preoperative physical activity on the incidence of paroxysmal atrial fibrillation. Am J Phys Med Rehabil 2011;90:308-15.

19. Cacciatore F, Belluomo Anello C, Ferrara N, et al. Determinants of prolonged intensive care unit stay after cardiac surgery in the elderly. Aging Clin Exp Res 2012;24:627-34.

20. Noyez L, Biemans I, Verkroost M, et al. Is a sedentary lifestyle an independent predictor for hospital and early mortality after elective cardiac surgery? Neth Heart $J$ 2013;21:439-45.

21. Min L, Mazzurco L, Gure TR, et al. Longitudinal functional recovery after geriatric cardiac surgery. J Surg Res 2015;194:25-33.

22. van Laar C, Kievit PC, Noyez L. Surgical aortic valve replacement in patients older than 75 years: is there really a quality of life benefit? Neth Heart J 2015;23:174-9.

23. van Poppel MN, Chinapaw MJ, Mokkink LB, et al. Physical activity questionnaires for adults: a systematic review of measurement properties. Sports Med Auckl NZ 2010;40:565-600.

24. Tremblay MS, Warburton DER, Janssen I, et al. New Canadian physical activity guidelines. Appl Physiol Nutr Metab 2011;36:36-46.

25. Physical activity guidelines Advisory Committee. Physical Activity Guidelines Advisory Committee Report. Washington, DC: U.S: Department of Health and Human Services, 2008.

26. Lee PH, Macfarlane DJ, Lam TH, et al. Validity of the International Physical Activity Questionnaire Short form (IPAQ-SF): a systematic review. Int J Behav Nutr Phys Act 2011;8:115.

27. Schmidt MD, Cleland VJ, Thomson RJ, et al. A comparison of subjective and objective measures of physical activity and fitness in identifying associations with cardiometabolic risk factors. Ann Epidemiol 2008;18:378-86.

28. Atienza AA, Moser RP, Perna F, et al. Self-reported and objectively measured activity related to biomarkers using NHANES. Med Sci Sports Exerc 2011;43:815-21.

29. Anderson L, Thompson DR, Oldridge N, et al. Exercise-based cardiac rehabilitation for coronary heart disease. Cochrane Database Syst Rev 2016;1:CD001800.

30. Goldfarb M, Drudi L, Almohammadi M, et al. Outcome Reporting in cardiac surgery trials: systematic review and critical appraisal. J Am Heart Assoc 2015;4:e002204.

31. Abah U, Dunne M, Cook A, et al. Does quality of life improve in octogenarians following cardiac surgery? A systematic review. BMJ Open 2015;5:e006904.

32. Karim MN, Reid CM, Tran L, et al. Missing Value Imputation improves mortality risk prediction following cardiac surgery: an investigation of an australian patient Cohort. Heart Lung Circ 2017;26:301-8. 